from the current inadequacies of the services caring for those with major mental disorder.

\section{Conclusion}

In the rush to adopt new fashions of practice it would be a pity if we lost the virtues of the past, particularly as not all areas may have problems in ensuring the assessment of offenders. Much more could be done to enable existing services to function better. Indeed unless resources are boundless the only pragmatic policy seems to be to foster a truly integrative service. Wherever possible assessment and treatment should be undertaken by the catchment area services. The costs of establishing parallel services to usurp these duties will be considerable. But if only some of the additional resources could be used to foster a greater interest in forensic psychiatry by all clinicians then the pool of potential workers would be enormous. This brief could easily be taken on by the regional forensic units. Interdisciplinary liaison can be improved by instigating informal and informative meetings, held on a regular basis and ideally occurring across a region. And with the help of academic departments in general psychiatry it should not be difficult to establish a culture in which forensic matters are held in high regard. One of our aims should be to make assessments of inmates as palatable as domiciliary visits to those in the community.

With so many experimental developments proceeding, it should be a priority to maintain an adequate control. Perhaps this could be akin to a 'reservation park', where general psychiatrists could be encouraged to practise the range of their skills, with assistance, as necessary, by fully trained specialist experts. I only wish that such a control could be in my own region!

\section{Acknowledgements}

I am indebted to all those who shared their experiences and thoughts with me, particularly all my colleagues in Wessex.

\section{References}

FAULK, M. (1985) Secure facilities in local psychiatric hospitals. In Secure Provision (ed. L. Gostin) London: Tavistock Publications.

Gibbens, T. C. N., SoOthill, K. L. \& Pope, P. J. (1977) Medical Remands in the Criminal Court. Oxford University Press.

GuNN, J. (1977) Management of the mentally abnormal offender: integrated or parallel. Proceedings of the Royal Society of Medicine, 70, 877-880.

Home OFfice (1990) Provision for Mentally Disordered Offenders. London: Home Office (circular 66/90).

JAMES, D. V. \& HAmilton, L. W. (1991) The Clerkenwell scheme: assessing efficacy and cost of a psychiatric liaison service to a magistrates' court. British Medical Journal, 303, 282-285.

Royal College of Psychiatrists (1988) The role, responsibilities and work of the consultant forensic psychiatrist. Bulletin of the Royal College of Psychiatrists, 12, 246-249.

\title{
A gravid glimpse at rapport
}

\author{
Grainne NeILsON, Psychiatric Registrar, Gartnavel Royal Hospital, 1055 Great \\ Western Road, Glasgow G12 0XH
}

As a psychiatric trainee I have often been intrigued by the respect that is accorded to pregnant women, deservingly or not, by the general public. People give up their seats on crowded trains and buses, they open doors, refuse to allow any heavy lifting and generally pamper the pregnant woman. As the months pass and the woman is transformed into a potential mother, attitudes towards her adjust, and she begins to be viewed in a different light. Certainly such was the case for me during my pregnancy and it was fascinating to observe the way in which the doctor-patient relationship changed as a result of my pregnant state.

I am employed at a large inner city psychiatric hospital which has many long-stay patients as well as those who are acutely psychiatrically unwell. Many of the older, chronically psychotic female long-stay patients (who ordinarily sit about the hospital grounds smoking and shouting abusive comments at 
passers-by) would approach me and tell me about their pregnancies and children. One lady even insisted on reading my palm to predict the sex of the baby (she was right!).

I worked until quite late on in my pregnancy (38 weeks) and on receiving days I began to correlate the patients' psychiatric diagnosis with how long it would take them to comment on my protruding abdomen.

Manic patients would tend to mention it immediately, pat my belly and then launch into a series of very personal questions regarding the baby's conception! Depressed patients would generally make no comment on their initial encounter with me, but I took their later enquiries about my preference for a boy or a girl as a fairly reliable sign that they were improving and taking more interest in people around them.

Patients with schizophrenia often seemed to incorporate pregnancy and babies into their symptomatology. One schizophrenic lad stopped suddenly mid-interview, mid-sentence and asked "Are you pregnant, Doctor?" When I replied that I was, he asked me in all sincerity, "Am I the father?" He felt he was experiencing such powerful bodily influence that somehow he had impregnated me without knowing. Thankfully this thought did not remain as part of his delusional system. Another acutely disturbed schizophrenic man threatened to pull a knife on me until he realised that I was pregnant and then offered to do a caesarean section! Luckily help was close at hand.

In psychotherapy, a female patient was able to talk for the first time about a termination she had had several years previously, and began to tackle very painful issues of loss and guilt surrounding this. It was interesting to observe this patient's ambivalence towards her own mother becoming evident in the transference as my impending motherhood drew closer.

Self-disclosure is a subject of some controversy in psychiatry but when the doctor is pregnant it is difficult for patients to deny the human component of their therapist and some degree of self-disclosure is therefore inevitable.

I am not sure whether pregnancy has such profound effects on the doctor-patient relationship in other branches of medicine, but certainly in psychiatry it enabled me to glimpse briefly into an aspect of my patients to which I might otherwise have never had access. For my part, perhaps now as a parent as well as a doctor I will have a more empathic understanding during my current placement in the Department of Child and Family Psychiatry.

\section{Correspondence}

\section{The management of empathy}

\section{DeAr Sirs}

The reforms of the NHS are altering our culture. The three Es of the business world-Effectiveness, Efficiency and Economy - are becoming the dominant value. In some places this is happening at the expense of a fourth $E$ - Empathy.

The task management has to create an environment in which efficient and effective treatment is provided with care and sensitivity. Patients who come for help expect a diagnosis which leads to effective treatment. But this is not all they want. They are in distress and so want care and concern. They want doctors and nurses who are willing to listen and able to understand how they are feeling. They need clinical staff who are empathic.
Empathy is a complex and demanding process which involves being in tune with the patient. It requires the clinician to put his own worries and concerns to one side in order to give his full attention to the patient: listening to patients, recognising their distress and responding to their emotional needs is taxing and demanding work. Although the capacity for empathy develops with training and experience it also needs a therapeutic setting in which sensitivity and understanding are possible. Privacy, quietness and uninterrupted time are essential. This means designing wards, clinics, consulting rooms etc in such a way that the necessary atmosphere of safety is created. Only then will patients be able to show their feelings.

Empathy needs management which involves attention to the corporate culture as well as to 